\title{
Multinomial logistic regression in workers' health
}

Luís M. Grilo, Helena L. Grilo, Sónia P. Gonçalves, and Ana Junça

Citation: AIP Conference Proceedings 1906, 110010 (2017); doi: 10.1063/1.5012392

View online: https://doi.org/10.1063/1.5012392

View Table of Contents: http://aip.scitation.org/toc/apc/1906/1

Published by the American Institute of Physics 


\title{
Multinomial Logistic Regression in Workers' Health
}

\author{
Luís M. Grilo ${ }^{1, a)}$, Helena L. Grilo ${ }^{2, b)}$, Sónia P. Gonçalves ${ }^{3,4, c)}$ and Ana Junça, $a^{3,4, d)}$ \\ ${ }^{1}$ Instituto Politécnico de Tomar, Departamento de Matemática e Física, Quinta do Contador - Estrada da Serra, \\ 2300-313, Tomar, and Centro de Matemática e Aplicações (CMA), FCT, UNL, Portugal. \\ ${ }^{2}$ Instituto Politécnico de Tomar, Centro de Sondagens e Estudos Estatísticos, Portugal. \\ ${ }^{3}$ Instituto Politécnico de Tomar, Departamento de Ciências Sociais, Portugal. \\ ${ }^{4}$ Instituto Universitário de Lisboa (ISCTE-IUL), Lisboa, Portugal. \\ a) Corresponding author: lgrilo@ipt.pt \\ b) helenagrilo56@gmail.com \\ c)sonia.goncalves@ipt.pt \\ d) analjsilva@ipt.pt
}

\begin{abstract}
In European countries, namely in Portugal, it is common to hear some people mentioning that they are exposed to excessive and continuous psychosocial stressors at work. This is increasing in diverse activity sectors, such as, the Services sector. A representative sample was collected from a Portuguese Services' organization, by applying a survey (internationally validated), which variables were measured in five ordered categories in Likert-type scale. A multinomial logistic regression model is used to estimate the probability of each category of the dependent variable general health perception where, among other independent variables, burnout appear as statistically significant.
\end{abstract}

\section{INTRODUCTION}

According to the European Agency for Safety and Health at Work, psychosocial risks and work-related stress are among the most challenging issues in occupational safety and health, once they have a significant impact on the individuals' health. The proportion of workers exposed to psychosocial stressors at work is growing. This has negative consequences for workers, organizations, and national economies, which may explain the increasing number of studies in this research field ([1, 2, 3, 5], among others).

The Copenhagen Psychosocial Questionnaire (COPSOQ) is an internationally validated method for the assessment of psychosocial risks and their impact on workers' health and wellbeing. It includes relevant dimensions according to several important theories on psychosocial factors in the workplace $[4,5]$. A short Portuguese version of COPSOQ (with 41 questions) was applied, in 2015, to a representative sample of workers of an important Portuguese organization of Services. We use 12 questions that belong to the 6 psychosocial dimensions (our independent variables) with epidemiological evidence for health, according to the medical community who are interested in identifying the possible causes and symptoms of some mental disturbs, such as burnout state, in order to assist the workers' treatment. We use multinomial logistic regression to estimate the probability of each of the 5 ordered categories (or levels) of the dependent variable general health perception (GHP), considering 4 quantitative independent variables (obtained as a mean of some of the 12 COPSOQ questions) and 2 qualitative variables as in Table 1. The dependent variable, with an approximately symmetric distribution, and the qualitative variables are coded in Table 2, which also includes the number of workers in each of the levels ( 3 have the highest numbers), marginal percentages and missing values.

In this study, Ordinal Regression is not considered because some assumptions are not verified, namely the test of parallel lines for the different link functions considered.

TABLE 1. Independent variables (quantitative and qualitative).

\begin{tabular}{|c|c|c|c|}
\hline 4 quantitative var. & COPSOQ questions & 2 qualitative var. & COPSOQ questions \\
\hline $\begin{array}{l}\text { Family-work } \\
\text { conflict }\end{array}$ & $\begin{array}{l}2 \text { questions about: to feel that the job requires a lot of } \\
\text { energy or time which ultimately affects negatively the } \\
\text { private life. }\end{array}$ & Sleeping troubles & $\begin{array}{l}\text { Woke up several times } \\
\text { during the night and then } \\
\text { could not fall asleep. }\end{array}$ \\
\hline Stress & 2 questions about: to be riled up or anxious. & Depressive symptoms & Sadness. \\
\hline Burnout & $\begin{array}{l}2 \text { questions about: emotionally and physically exhausted. } \\
4 \text { questions about: to have been the target of insults and }\end{array}$ & & \\
\hline Offensive behaviour & $\begin{array}{l}\text { verbal provocations, have been exposed to physical } \\
\text { violence, unwanted sexual harassment. }\end{array}$ & & \\
\hline
\end{tabular}

Proceedings of the International Conference of Computational Methods in Sciences and Engineering 2017 (ICCMSE-2017) AIP Conf. Proc. 1906, 110010-1-110010-5; https://doi.org/10.1063/1.5012392 Published by AIP Publishing. 978-0-7354-1596-6/\$30.00 
TABLE 2. Case Processing Summary.

\begin{tabular}{llcc}
\hline & & N & Marginal Percentage \\
\hline & 1 - deficient & 235 & $4.7 \%$ \\
GHP: & 2 - reasonable & 1223 & $24.5 \%$ \\
(dependent variable) & $\mathbf{3}$ - good & 1965 & $39.4 \%$ \\
& $\mathbf{4}$ - very good & 1207 & $24.2 \%$ \\
& $\mathbf{5}$ - excellent & 355 & $7.1 \%$ \\
\hline & $\mathbf{1}$ - nothing & 832 & $16.7 \%$ \\
Sleeping troubles: & $\mathbf{2}$ - little & 1043 & $20.9 \%$ \\
(independent variable) & $\mathbf{3}$ - moderately & 1556 & $31.2 \%$ \\
& $\mathbf{4}$ - very & 1338 & $26.8 \%$ \\
& $\mathbf{5}$ - extremely & 216 & $4.3 \%$ \\
\hline & $\mathbf{1}$ - nothing & 800 & $16.0 \%$ \\
Depressive symptoms: & $\mathbf{2}$ - little & 1333 & $26.7 \%$ \\
(independent variable) & $\mathbf{3}$ - moderately & 1783 & $35.8 \%$ \\
& $\mathbf{4}$ - very & 858 & $17.2 \%$ \\
\hline Valid & $\mathbf{5}$ - extremely & 211 & $4.2 \%$ \\
Missing & & 4985 & $100.0 \%$ \\
Total & 199 & \\
Subpopulation & & 5184 & \\
\hline a. The dependent variable has only one value observed in $2017(75.7 \%)$ subpopulations.
\end{tabular}

\section{The multinomial logistic regression model}

Multinomial logistic regression is a predictive model once it is used to predict the probabilities of the different possible outcomes of a categorically distributed dependent variable, given a set of independent variables (6 in our study). Let $Y$ be the dependent variable $G H P$, with $K=5$ categories, then the multinomial regression consists of a system of 5 logistic models which, after being standardized for the reference category of $Y$ (in this case study we consider the first category "1-deficient"), allow us to compute the probability of $Y$ taking the value of each one of the 5 categories. In matrix notation, where $\mathbf{X}$ is the matrix of the independent variables and $\boldsymbol{\beta}$ the vector of the coefficients $[6,7]$,

$$
P(Y=1 \mid \mathbf{X})=\frac{1}{1+\sum_{k=2}^{5} e^{\mathbf{X} \beta_{k}}}, \quad P(Y=2 \mid \mathbf{X})=\frac{e^{\mathbf{X} \beta_{2}}}{1+\sum_{k=2}^{5} e^{\mathbf{X}_{k}}}, \ldots, \quad P(Y=5 \mid \mathbf{X})=\frac{e^{\mathbf{X}_{5}}}{1+\sum_{k=2}^{5} e^{\mathbf{X}_{\mathrm{k}}}} .
$$

The $K-1=4$ odds of each category of $Y$, relatively to the reference category, are given by,

$$
\frac{P(Y=2 \mid \mathbf{X})}{P(Y=1 \mid \mathbf{X})}=e^{\mathbf{x}_{2}}, \quad \frac{P(Y=3 \mid \mathbf{X})}{P(Y=1 \mid \mathbf{X})}=e^{\mathbf{x} \boldsymbol{\beta}_{3}}, \quad \frac{P(Y=4 \mid \mathbf{X})}{P(Y=1 \mid \mathbf{X})}=e^{\mathbf{x}_{\mathbf{\beta}_{4}}}, \quad \frac{P(Y=5 \mid \mathbf{X})}{P(Y=1 \mid \mathbf{X})}=e^{\mathbf{x}_{\beta_{5}}}
$$

and logarithmizing the two members of the equality we obtain $4 \log$ odds, relatively to the reference category, of $Y$ that constitute the multi-equation model,

$$
\ln \frac{P(Y=2 \mid \mathbf{X})}{P(Y=1 \mid \mathbf{X})}=\mathbf{X} \boldsymbol{\beta}_{2}, \quad \ln \frac{P(Y=3 \mid \mathbf{X})}{P(Y=1 \mid \mathbf{X})}=\mathbf{X} \boldsymbol{\beta}_{3}, \quad \ln \frac{P(Y=4 \mid \mathbf{X})}{P(Y=1 \mid \mathbf{X})}=\mathbf{X} \boldsymbol{\beta}_{4}, \quad \ln \frac{P(Y=5 \mid \mathbf{X})}{P(Y=1 \mid \mathbf{X})}=\mathbf{X} \boldsymbol{\beta}_{5} .
$$

Thus, the logit for each non-reference category against the reference category (that it is the "standard" category to which the others will be naturally compared) depends on the values of the explanatory variables. The model is iteratively adjusted with the maximum likelihood method.

\section{ANALYSIS AND RESULTS}

After adjusting the multinomial logistic regression model, it is necessary to determine whether it reasonably approximates the behaviour of the available data. The likelihood ratio test of the final model (with all the independent variables) against the null model (only with a constant and where all the parameter coefficients are 0) and the chi-square statistic, as the difference between the $-2 \log$-likelihoods (LL) of the null and final model, are presented in Table 3 . Once the $p$-value $=0.000$ (less than the usual significance levels, namely 0.01 ), we can conclude that the final model is outperforming the null, i.e. the adjusted model is statistically significant. Therefore, there is at least one 
independent variable of significant influence on the workers' GHP. Consistent with this are the smaller values of AIC (Akaike information criterion) and BIC (Bayesian information criterion) in the final model.

TABLE 3. Model Fitting Information (Model Fitting Criteria and Likelihood Ratio Tests).

\begin{tabular}{lcccccc}
\hline \multirow{2}{*}{ Model } & \multicolumn{3}{c}{ Model Fitting Criteria } & \multicolumn{3}{c}{ Likelihood Ratio Tests } \\
\cline { 2 - 7 } & AIC & BIC & $\mathbf{- 2}$ Log-Likelihood Chi-Square & df & p-value \\
\hline Intercept Only 10511.551 & 10537.608 & 10503.551 & & & \\
Final & 9182.425 & 9495.106 & 9086.425 & 1417.126 & 44 & 0.000 \\
\hline
\end{tabular}

About the validity of the goodness-of-fit tests (Pearson and Deviance, available in the IBM SPSS, used in this study) we should note that there are 9745 cells (i.e., dependent variable levels by subpopulations) with zero frequencies. Thus, because there are many empty cells (73.1\%), we cannot safely use the results of these tests.

To select the "best" predictors to include in the model we use the automatic stepwise method Forward Entry, which starts with a model that only includes the intercept and where the final model should only include important predictors. In Table 4 we have the likelihood ratio tests, which check the contribution of each effect to the model. For each one, the -2LL is computed for the reduced model (that is, a model without the effect) and the chi-square statistic is the difference between the -2LL of the reduced model from Table 4 and the final model reported in Table 3.

The results of the likelihood ratio tests for each of the independent variables indicate that the variables family-work conflict, stress, depressive symptoms, sleeping troubles and burnout have a statistically significant effect (all p-values are less than 0.01) under the logit of the probability of having a $G H P$, at least "2-reasonable", relatively to the reference category "1-deficient". The variable offensive behaviour does not appear as statistically significant. We should note that the intercept cannot be tested in this model because removing it simply causes one of the previously redundant factor levels to become non-redundant.

TABLE 4. The likelihood ratio tests (Model Fitting Criteria and Likelihood Ratio Tests).

\begin{tabular}{|c|c|c|c|c|c|c|}
\hline \multirow[b]{2}{*}{ Effect } & \multicolumn{3}{|c|}{ Model Fitting Criteria } & \multicolumn{3}{|c|}{ Likelihood Ratio Tests } \\
\hline & $\begin{array}{c}\text { AIC of Reduced } \\
\text { Model }\end{array}$ & $\begin{array}{c}\text { BIC of Reduce } \\
\text { Model }\end{array}$ & $\begin{array}{l}\text { d-2 Log-Likelihood } \\
\text { of Reduced Model }\end{array}$ & Chi-Square & df & p-value \\
\hline Intercept & 9182.425 & 9495.106 & $9086.425^{\mathrm{a}}$ & 0.000 & . & \\
\hline Family-work conflict & 9188.928 & 9475.552 & 9100.928 & 14.503 & 4 & 0.006 \\
\hline Stress & 9190.852 & 9477.476 & 9102.852 & 16.427 & 4 & 0.002 \\
\hline Depressive symptoms & 9294.722 & 9503.176 & 9230.722 & 144.297 & 16 & 0.000 \\
\hline Sleeping troubles & 9273.672 & 9482.126 & 9209.672 & 123.248 & 16 & 0.000 \\
\hline Burnout & 9235.001 & 9521.625 & 9147.001 & 60.576 & 4 & 0.000 \\
\hline
\end{tabular}

The probability of observing each dependent variable category is a function of the predictors given by the estimated parameters (denoted by B in Table A1, Appendix I), through equations in (1). The estimated parameters of the multinomial model summarize the effect of each predictor, where the ratio between each coefficient and its standard error, squared, equals the Wald statistic. The odd ratios (OR) for each category of the dependent variable, relative to the reference category ("1-deficient"), are available for each of the independent variables in the Exp(B) column of Table A1. Parameters with significant negative/positive coefficients decrease/increase the likelihood of that response category with respect to the reference category. When B is positive/negative then $\operatorname{Exp}(\mathrm{B})$ is higher/smaller than 1, which means that $\mathrm{OR}$ is $\operatorname{Exp}(\mathrm{B})$ to 1 , thus the chance of having a certain level of $G H P$ is $|1-\operatorname{Exp}(\mathrm{B})| \times 100 \%$ higher/lower than the reference category (or, $\operatorname{Exp}(B)$ times higher/lower in that level than in level "1-deficient"). Note that, the parameters associated with the last level of each factor is redundant given the intercept term.

For "3-good" GHP, which is the category with the highest number of individuals, only family-work conflict and stress are not statistically significant, compared to the reference category. However, all levels of depressive symptoms and sleeping troubles as well as the burnout are statistically significant (the p-values of the Wald statistic are less than 0.05 , then the null hypotheses of the parameters being equal to zero are rejected). Thus, we can say that almost all the independent variables allow to distinguish the probabilities of the category "3-good" compared to the category "1deficient", but the same situation does not occur when we compare the coefficients of the category "2-reasonable" (where the majority of the independent variables are not statically significant) considering the reference category.

Passing from "1-deficient" to "5-excellent" GHP is significantly affected by burnout $(\mathrm{B}=-0.826$, $\mathrm{p}$-value $=0.000$, $\mathrm{OR}=0.438$ ), whenever we pass from one burnout level to the next/previous level, the chances of having excellent $G H P$ decrease/increase $|1-0.438| \times 100=56.2 \%$ (comparing with "1-deficient" $G H P$ ). Considering the independent 
qualitative variable depressive symptoms, workers with "1-nothing" present a higher probability of "5-excellent" GHP than those with "5-extremely". Having "1-nothing" in relation to having "5-extremely" depressive symptoms increases the chance of "5-excellent" relatively to " 1 -deficient" GHP in $|1-4.825| \times 100=382.5 \%$ (Table A1, Appendix I).

\section{Classification Results}

We use multinomial logistic regression to classify individuals based on values of a set of predictor variables. The outputs of Table A1 (Appendix I) allow us to compute the probability of each individual belonging to each one of the dependent variable categories. For each individual, the predicted response category is chosen by selecting the one with the highest model-predicted probability. Table 5 shows the practical results of using this model, where cells on/off the diagonal are correct/incorrect predictions. Considering the dependent variable GHP with the five categories from "1-deficient" to "5-excellent" we can say, for example, that 1366 of the 1965 workers who chose "3-good" are classified correctly (approximately 69.5\%). Overall, $45.2 \%$ of the individuals are classified correctly and the proportional percentage, based on Table 2 , is $\left(0.047^{2}+0.245^{2}+0.394^{2}+0.242^{2}+0.071^{2}\right) \times 100 \% \approx 28.1 \%$. Thus, the model gives almost twice the correct classifications than would be obtained by mere chance. This compares favourably to the "null", or intercept-only model, which classifies all cases as the modal category.

TABLE 5. Classification (Observed versus Predicted).

\begin{tabular}{lcccccc}
\hline \multirow{2}{*}{ Observed } & \multicolumn{7}{c}{ Predicted } \\
\cline { 2 - 7 } & $1-$ deficient & 2-reasonable & $3-$ good & $4-$ very good & 5 - excellent & Percent Correct \\
\hline 1- deficient & $\mathbf{2 1}$ & 119 & 75 & 20 & 0 & $8.9 \%$ \\
2- reasonable & 16 & $\mathbf{4 2 0}$ & 695 & 92 & 0 & $34.3 \%$ \\
3 - good & 2 & 294 & $\mathbf{1 3 6 6}$ & 303 & 0 & $69.5 \%$ \\
4- very good & 1 & 81 & 677 & $\mathbf{4 4 8}$ & 0 & $37.1 \%$ \\
5 - excellent & 1 & 14 & 158 & 182 & $\mathbf{0}$ & $0.0 \%$ \\
\hline Overall Percentage & $0.8 \%$ & $18.6 \%$ & $59.6 \%$ & $21.0 \%$ & $0.0 \%$ & $\mathbf{4 5 . 2 \%}$ \\
\hline
\end{tabular}

\section{CONCLUSIONS AND FINAL REMARKS}

Using the multinomial logistic regression procedure, we have constructed a model for predicting workers' opinions about their GHP. According to the adjusted model, passing from the reference category ("1-deficient") to any of the other categories is not significantly affected by stress, but it is affected in a statistically significant way by the variables burnout and some levels of depressive symptoms and sleeping troubles. In general, the probability of having higher levels of GHP increases for the same decrease of level in these variables.

This case study also allows us to obtain the number of workers classified correctly in each category of the dependent variable.

\section{ACKNOWLEDGMENTS}

This work was partially supported by the Fundação para a Ciência e a Tecnologia (Portuguese Foundation for Science and Technology) through the project UID/MAT/00297/2013 (Centro de Matemática e Aplicações).

\section{REFERENCES}

1. A. Bakker, E. Demerouti and A. I. Sanz-Vergel, Annual Review of Organizational Psychology and Organizational Behaviour, 1(1), 140114155134003 (2014).

2. C. Maslach and M. P. Leiter, Journal of Applied Psychology 93: 498-512 (2008).

3. M. Stehlík, Ch. Helpersdorfer, P. Hermann, J. Šupina, L. M. Grilo, J. P. Maidana, F. Fuders and S. Stehlíková, Information Sciences 394-395, 246-272 (2017).

4. H. V. Neto, J. Areosa and P. Arezes, Manual sobre Riscos Psicossociais no Trabalho (Civeri Publishing, Col. RICOT, 2014).

5. T. S. Kristensen, M. Borritz, E. Villadsen and K. B. Christensen, Work \& Stress 19: 192-207 (2005).

6. D. W. Hosmer and S. Lemeshow, Applied Logistic Regression ( $2^{\text {nd }}$ Edition, John Wiley \& Sons, New York, 2000).

7. W. H. Greene, Econometric Analysis. (Seventh ed., Boston: Pearson Education. 803-806, 2012). 


\section{APPENDIX I}

TABLE A1. The estimated parameters of the Multinomial Model.

\begin{tabular}{|c|c|c|c|c|c|c|c|c|c|}
\hline & \multirow{2}{*}{$G H P:^{a}$} & \multirow{2}{*}{ B } & \multirow{2}{*}{\multicolumn{2}{|c|}{ Std. Error Wald }} & \multirow{2}{*}{ df } & \multirow{2}{*}{ p-value } & \multirow{2}{*}{$\operatorname{Exp}(B)$} & \multicolumn{2}{|c|}{ 95\% Confidence Interval for $\operatorname{Exp}(B)$} \\
\hline & & & & & & & & Lower Bound & Upper Bound \\
\hline \multirow{14}{*}{ 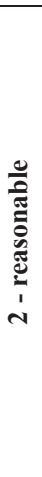 } & Intercept & 0.452 & 0.768 & 0.346 & 1 & 0.557 & & & \\
\hline & Family-work conflict & -0.085 & 0.091 & 0.871 & 1 & 0.351 & 0.919 & 0.769 & 1.098 \\
\hline & Stress & 0.166 & 0.139 & 1.427 & 1 & 0.232 & 1.181 & 0.899 & 1.551 \\
\hline & {$[$ Depressive symptoms $=1]$} & 0.781 & 0.421 & 3.440 & 1 & 0.064 & 2.184 & 0.957 & 4.984 \\
\hline & [Depressive symptoms $=2]$ & 1.559 & 0.365 & 18.248 & 1 & 0.000 & 4.752 & 2.324 & 9.715 \\
\hline & {$[$ Depressive symptoms $=3]$} & 1.565 & 0.271 & 33.262 & 1 & 0.000 & 4.784 & 2.810 & 8.143 \\
\hline & {$[$ Depressive symptoms $=4]$} & 1.004 & 0.229 & 19.310 & 1 & 0.000 & 2.730 & 1.744 & 4.272 \\
\hline & {$[$ Depressive symptoms $=5]$} & $0^{\mathrm{b}}$ & . & . & . & . & . & . & . \\
\hline & {$[$ Sleeping troubles $=1]$} & 0.704 & 0.405 & 3.028 & 1 & 0.082 & 2.023 & 0.915 & 4.472 \\
\hline & {$[$ Sleeping troubles $=2]$} & 1.161 & 0.371 & 9.812 & 1 & 0.002 & 3.193 & 1.544 & 6.602 \\
\hline & {$[$ Sleeping troubles $=3]$} & 0.663 & 0.270 & 6.011 & 1 & 0.014 & 1.941 & 1.142 & 3.297 \\
\hline & {$[$ Sleeping troubles $=4]$} & 0.402 & 0.228 & 3.110 & 1 & 0.078 & 1.495 & 0.956 & 2.337 \\
\hline & {$[$ Sleeping troubles $=5]$} & $0^{\mathrm{b}}$ & . & . & . & . & . & . & . \\
\hline & Burnout & -0.182 & 0.150 & 1.472 & 1 & 0.225 & 0.834 & 0.622 & 1.118 \\
\hline \multirow{14}{*}{ 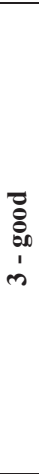 } & Intercept & 1.643 & 0.768 & 4.579 & 1 & 0.032 & & & \\
\hline & Family-work conflict & -0.168 & 0.090 & 3.456 & 1 & 0.063 & 0.845 & 0.708 & 1.009 \\
\hline & Stress & 0.203 & 0.138 & 2.157 & 1 & 0.142 & 1.225 & 0.934 & 1.605 \\
\hline & {$[$ Depressive symptoms $=1]$} & 1.147 & 0.423 & 7.343 & 1 & 0.007 & 3.148 & 1.373 & 7.214 \\
\hline & [Depressive symptoms $=2]$ & 2.288 & 0.371 & 37.920 & 1 & 0.000 & 9.852 & 4.757 & 20.404 \\
\hline & {$[$ Depressive symptoms $=3]$} & 1.987 & 0.286 & 48.142 & 1 & 0.000 & 7.295 & 4.161 & 12.788 \\
\hline & {$[$ Depressive symptoms $=4]$} & 1.064 & 0.251 & 17.991 & 1 & 0.000 & 2.899 & 1.773 & 4.740 \\
\hline & {$[$ Depressive symptoms $=5]$} & $0^{\mathrm{b}}$ & . & . & . & . & . & . & . \\
\hline & {$[$ Sleeping troubles $=1]$} & 1.216 & 0.407 & 8.917 & 1 & 0.003 & 3.372 & 1.518 & 7.489 \\
\hline & {$[$ Sleeping troubles $=2]$} & 1.642 & 0.378 & 18.872 & 1 & 0.000 & 5.163 & 2.462 & 10.829 \\
\hline & {$[$ Sleeping troubles $=3]$} & 1.250 & 0.284 & 19.415 & 1 & 0.000 & 3.491 & 2.002 & 6.088 \\
\hline & {$[$ Sleeping troubles $=4]$} & 0.537 & 0.248 & 4.684 & 1 & 0.030 & 1.710 & 1.052 & 2.781 \\
\hline & {$[$ Sleeping troubles $=5]$} & $0^{\mathrm{b}}$ & . & . & . & . & . & . & . \\
\hline & Burnout & -0.579 & 0.148 & 15.281 & 1 & 0.000 & 0.561 & 0.419 & 0.749 \\
\hline \multirow{14}{*}{ 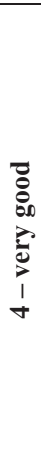 } & Intercept & 1.977 & 0.834 & 5.619 & 1 & 0.018 & & & \\
\hline & Family-work conflict & -0.149 & 0.095 & 2.465 & 1 & 0.116 & 0.862 & 0.716 & 1.038 \\
\hline & Stress & -0.059 & 0.144 & 0.166 & 1 & 0.683 & 0.943 & 0.711 & 1.250 \\
\hline & {$[$ Depressive symptoms $=1]$} & 1.526 & 0.483 & 10.005 & 1 & 0.002 & 4.601 & 1.787 & 11.845 \\
\hline & {$[$ Depressive symptoms $=2]$} & 2.489 & 0.436 & 32.524 & 1 & 0.000 & 12.044 & 5.121 & 28.326 \\
\hline & {$[$ Depressive symptoms $=3]$} & 1.775 & 0.367 & 23.411 & 1 & 0.000 & 5.901 & 2.875 & 12.110 \\
\hline & {$[$ Depressive symptoms $=4]$} & 0.819 & 0.347 & 5.576 & 1 & 0.018 & 2.268 & 1.149 & 4.475 \\
\hline & {$[$ Depressive symptoms $=5]$} & $0^{\mathrm{b}}$ & . & . & . & . & . & . & . \\
\hline & {$[$ Sleeping troubles $=1]$} & 1.663 & 0.456 & 13.305 & 1 & 0.000 & 5.277 & 2.159 & 12.898 \\
\hline & {$[$ Sleeping troubles $=2]$} & 1.968 & 0.431 & 20.877 & 1 & 0.000 & 7.159 & 3.077 & 16.654 \\
\hline & {$[$ Sleeping troubles $=3]$} & 1.265 & 0.352 & 12.936 & 1 & 0.000 & 3.545 & 1.779 & 7.065 \\
\hline & {$[$ Sleeping troubles $=4]$} & 0.383 & 0.327 & 1.376 & 1 & 0.241 & 1.467 & 0.773 & 2.784 \\
\hline & {$[$ Sleeping troubles $=5]$} & $0^{\mathrm{b}}$ & . & . & . & . & . & . & . \\
\hline & Burnout & -0.691 & 0.153 & 20.326 & 1 & 0.000 & 0.501 & 0.371 & 0.677 \\
\hline \multirow{14}{*}{ 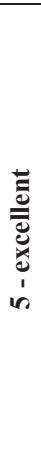 } & Intercept & 2.382 & 1.024 & 5.413 & 1 & 0.020 & & & \\
\hline & Family-work conflict & -0.354 & 0.111 & 10.145 & 1 & 0.001 & 0.702 & 0.565 & 0.873 \\
\hline & Stress & -0.002 & 0.168 & 0.000 & 1 & 0.988 & 0.998 & 0.718 & 1.386 \\
\hline & {$[$ Depressive symptoms $=1]$} & 1.574 & 0.682 & 5.327 & 1 & 0.021 & 4.825 & 1.268 & 18.365 \\
\hline & [Depressive symptoms $=2]$ & 2.275 & 0.640 & 12.622 & 1 & 0.000 & 9.730 & 2.773 & 34.136 \\
\hline & [Depressive symptoms $=3]$ & 1.586 & 0.591 & 7.201 & 1 & 0.007 & 4.886 & 1.534 & 15.568 \\
\hline & {$[$ Depressive symptoms $=4]$} & 0.484 & 0.606 & 0.637 & 1 & 0.425 & 1.622 & 0.495 & 5.322 \\
\hline & {$[$ Depressive symptoms $=5]$} & $0^{\mathrm{b}}$ & . & . & . & . & . & . & . \\
\hline & {$[$ Sleeping troubles $=1]$} & 1.190 & 0.550 & 4.690 & 1 & 0.030 & 3.288 & 1.120 & 9.656 \\
\hline & {$[$ Sleeping troubles $=2]$} & 1.105 & 0.529 & 4.361 & 1 & 0.037 & 3.018 & 1.070 & 8.511 \\
\hline & {$[$ Sleeping troubles $=3]$} & 0.242 & 0.466 & 0.269 & 1 & 0.604 & 1.274 & 0.511 & 3.177 \\
\hline & {$[$ Sleeping troubles $=4]$} & -0.602 & 0.460 & 1.714 & 1 & 0.190 & 0.548 & 0.222 & 1.349 \\
\hline & {$[$ Sleeping troubles $=5]$} & $0^{\mathrm{b}}$ & . & . & . & . & . & . & . \\
\hline & Burnout & -0.826 & 0.174 & 22.509 & 1 & 0.000 & 0.438 & 0.311 & 0.616 \\
\hline
\end{tabular}

b. This parameter is set to zero because it is redundant. 